\title{
Erratum: Possible nonequilibrium imprint in the cosmic background at low frequencies [Phys. Rev. Research 2, 013210 (2020)]
}

Marco Baiesi, Carlo Burigana, Livia Conti $\odot$, Gianmaria Falasco, Christian Maes, Lamberto Rondoni, and Tiziana Trombetti

(Received 18 November 2020; published 11 December 2020)

DOI: 10.1103/PhysRevResearch.2.049001

Figure 2 in the published version of our paper is wrong because of a mere exchange of files during the work finalization. In this erratum we provide a correct version where a better comparison between the functions considered for the diffusivity $D(v)$ is also shown. This figure version includes the right scale for $D(v)$ in panel (a) and the corrected functions for the dimensionless photon occupation number $n_{s}(v)$ in panel (b).

All the concepts and conclusions of the original paper remain valid and unaffected.
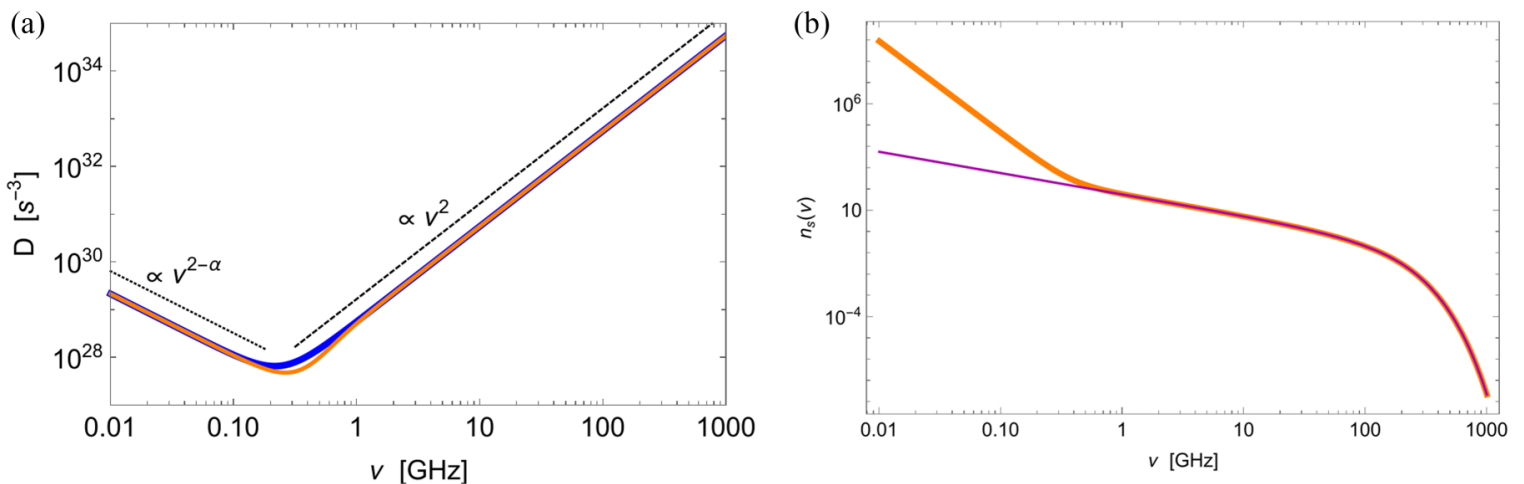

FIG. 2. For the parameters resulting from the fit of the data set in column (a) of Table I with both $\alpha$ and $v_{0}$ as free parameters. (a) The diffusivity $D(v)$ corresponding to the approximation in (16) adopted in the fit (orange solid line) and, for comparison, the one given in (13) (blue solid line); the dotted line represents a power-law $\propto v^{2-\alpha}$ whereas the dashed one $\propto v^{2}$. (b) Plots of the dimensionless photon occupation number $n_{s}(v)$ from and (15) and (16) (orange thick solid line) compared with the curve associated with the Planck law at $T^{*}$ (magenta solid line). 